\title{
Avaliação da linguagem em crianças com deficiência visual: Adaptação do Teste
}

\author{
ABFW \\ Language assessment of children with visual impairment: Adaptation of ABFW \\ Test
}

\author{
Eline Silva Rodrigues***, Anabela Cruz-Santos **, Jáima Pinheiro de Oliveira*** \\ *Instituto Benjamin Constant, Brasil, **Instituto de Educação, CIEd, Universidade do Minho ***Universidade Estadual Paulista \\ Júlio de Mesquita Filho
}

\begin{abstract}
Resumen
Este artigo apresenta resultados preliminares de um estudo piloto cujo objetivo geral foi adaptar as Provas de Fonologia, Vocabulário e Pragmática do ABFW Teste de Linguagem Infantil para verificar sua aplicabilidade e eficácia na avaliação em crianças cegas e com baixa visão na faixa etária dos 3 aos 7 anos, no Estado do Rio de Janeiro. Conclui-se que a versão adaptada do ABFW possibilita a avaliação da linguagem em crianças com deficiência visual, tendo sido eficaz em termos de acessibilidade para essa população. Serão apresentadas as adaptações realizadas nas provas para a deficiência visual. Palavras-chave: avaliação, linguagem, deficiência visual, Teste ABFW
\end{abstract}

\begin{abstract}
This paper presents preliminary results of a research whose main objective was to adapt the Tests of Phonology, Vocabulary and Pragmatics of the ABFW Infant Language Test to verify its applicability and effectiveness in assessment of blind and low vision children in the age group from 3 to 7 years, in Rio de Janeiro. It is concluded that the adapted version of ABFW allows the assessment of the language in children with visual impairment and has been effective in terms of accessibility for this population. All adaptations of the tests for visual impairment will be presented.

Keywords: assessment, language, visual impairment, Test ABFW
\end{abstract}

A linguagem é um marcador fundamental no desenvolvimento global infantil. O desenvolvimento atípico da linguagem pode provocar dificuldades que incluem desde aspectos relacionados à aprendizagem da leitura e escrita até a limitações das competências sociais (Rigolet, 2006; Sim-Sim, 1998).

A avaliação da linguagem em crianças com deficiência visual (DV) possibilita o conhecimento de suas competências linguísticas e a identificação de possíveis problemas na linguagem, dados cruciais para a intervenção atempada e eficaz.

Porém, a deficiência visual pode influenciar a performance da criança em testes não adaptados. A adoção de instrumentos inadequados na avaliação da criança com deficiência visual pode produzir resultados que não refletem seu verdadeiro estágio de desenvolvimento (Bishop, 1998; Mosca, Kritzinger \& Van der Linde, 2015; Perez-Pereira, 2014).

Portanto, assegurar a acessibilidade tanto nos instrumentos quanto nos procedimentos é um aspecto fundamental no planeamento da avaliação da linguagem em crianças cegas ou com baixa visão.

Dito isto, qualquer que seja a dimensão da avaliação da linguagem de crianças com DV, esta demanda a utilização de instrumentos adaptados e validados às suas especificidades (Mcconachie \& Moore, 1994; Van der Vaan, Vervloed, Knoors, \& Verhoeven, 2013).

No contexto brasileiro, existe uma carência de instrumentos validados e estandardizados para crianças cegas ou com baixa visão em idades pré-escolar e escolar. Estes fatores podem dificultar o processo de avaliação e o diagnóstico precoce de possíveis perturbações de linguagem nesta população.

Ademais, ganha particular relevo não só a complexidade subjacente às concepções e práticas de avaliação da linguagem em crianças com DV, como também, a necessidade de aprofundar formas e instrumentos de avaliação ajustados à diversidade desse grupo, especialmente nos primeiros anos de vida (Silver, 2003).

Considerando o estado da investigação neste domínio, a adaptação de um teste estandardizado apresenta vantagens teórico-metodológicas por ser uma abordagem prática que oferece a possibilidade de usar como base um modelo, no caso do Teste ABFW (Andrade, C., BefiLopes, D., Fernandes, F., \& Wertzner, H., 2011) já estandardizado e amplamente utilizado no Brasil, para crianças com desenvolvimento típico.

\section{Método}

\section{Participantes}

Este estudo foi realizado de setembro a dezembro de 2016, no Instituto Benjamin Constant (IBC), Centro de Referência Nacional na Educação de Pessoas com Deficiência visual no Brasil. 
A pesquisa foi aprovada pelo Comitê de Ética responsável sob o Protocolo de Pesquisa 095145/2016 e os responsáveis consentiram na participação dos sujeitos na pesquisa, assinando o Termo de Consentimento Livre e Esclarecido

Conforme os critérios metodológicos previamente definidos na pesquisa, para etapa do estudo piloto, foram selecionados 10 crianças com DV, com idades entre os 3 e 7 anos, sendo $50 \%$ do gênero feminino e $50 \%$ do gênero masculino.

As crianças foram organizadas em dois grupos conforme a condição visual: cinco crianças cegas e cinco crianças com baixa visão.

\section{Instrumentos}

Indicado para avaliar a linguagem de crianças com idades entre os 2 e 12 anos nas áreas de Fonologia, Vocabulário, Fluência e Pragmática, o ABFW é um teste desenvolvido para usuários do Português Brasileiro.

Compõe-se de quatro partes: Fonologia (Parte A), Vocabulário (Parte B), Fluência (Parte C) e Pragmática (Parte D), constituindo-se em instrumento de referência para o diagnóstico e planejamento de condutas de terapia dos distúrbios da comunicação.

No design original do ABFW o principal estímulo utilizado nas provas são figuras coloridas impressas em forma de prancha que medem $12 \mathrm{~cm}$ x $21 \mathrm{~cm}$ (Fichário ABFW).

Nossa pesquisa centrou-se na adaptação de três das quatro provas que compõem o teste, sendo a Prova de Fonologia (Parte A), a do Vocabulário (Parte B) e de Pragmática (Parte D).

\section{Procedimento}

Dada as especificidades do grupo das crianças cegas e do grupo das crianças com baixa visão - o processo de adaptação demandou uma criteriosa avaliação da versão original do ABFW.

Foram analisados todos os aspectos referentes ao protocolo de aplicação das três provas, a natureza estímulos utilizados dentre outros itens, para a planificação das propostas de adaptação para os dois grupos.

Este primeiro estudo viabilizou o levantamento das demandas iniciais de acessibilidade tanto para as crianças cegas quanto para as com baixa visão.

Posteriormente, tendo em vista o desenvolvimento de um formato acessível para crianças com DV, foram definidos os principais componentes das três provas do ABFW que requeriam adaptações:

1. Adequação dos procedimentos de aplicação das provas;

2. Adaptação ou substituição dos estímulos;

3. Adequação do ambiente

4. Adequação do tempo

Após os ajustes iniciais foi efetuado o segundo estudo, utilizando a amostra de 10 crianças com DV. Todas as intervenções foram gravadas em áudio e vídeo no setor de Fonoaudiologia do IBC. As filmagens foram realizadas individualmente, conforme indicação do protocolo original do teste.

\section{Resultados}

As três provas do ABFW foram analisadas, adaptadas e aplicadas em um estudo-piloto. Os resultados pertinentes a cada prova serão apresentados a seguir, observando a especificidade de cada grupo.

A Prova de Imitação - Fonologia (a criança deve repetir 39 vocábulos) não necessitou de adaptação, visto que a atividade proposta no formato original pode ser perfeitamente executada pelas crianças com deficiência DV.

Em contrapartida, em virtude das características, objetivos e materiais, as Provas de Nomeação Fonologia, a Prova de Vocabulário e a Prova de Pragmática demandam adaptação.

\section{Adaptação dos estímulos - Figuras \\ 1.Crianças cegas}

- Prova de Nomeação - Fonologia e Prova de Vocabulário (a criança deve nomear as figuras): as 152 figuras do fichário original foram substituídas por objetos reais, miniaturas, maquete multissensorial, figuras táteis, alimentos in natura e sons típicos relativos aos vocábulos da prova.

$\mathrm{Na}$ seleção das miniaturas, apesar das diferenças em relação objeto real como tamanho, peso, textura e outros aspectos, primamos por aqueles modelos que apresentavam melhor experiência tátil para a criança cega.

A utilização de miniaturas e objetos reais para a execução da Prova de Nomeação e os dos campos conceituais "Vestuário, Animais, Meios de Transporte, Móveis e Utensílios, Profissões, Brinquedos e Instrumentos Musicais" da Prova de Vocabulário demonstrou ser uma adaptação válida.

$\mathrm{O}$ exame da performance das crianças nessas provas, com a nomeação correta dos estímulos, indica um grau satisfatório de acessibilidade do material selecionado.

Para o campo conceitual "Alimentos", utilizamos alimentos in natura. Desse modo garantimos o acesso ao cheiro, textura, temperatura, dentre outras características de cada alimento listado no teste. Os alimentos foram apresentados inteiros, fracionados ou em pequenas porções.

As 12 figuras do campo conceitual "Locais" foram representadas em uma maquete multissensorial (ver Figura 1). O modelo foi elaborado considerando a exploração através dos sentidos remanescentes, ou seja, a criança pode tocar o sino da igreja, sentir a água do rio, o cheiro do jardim, movimentar o carro na rua. 


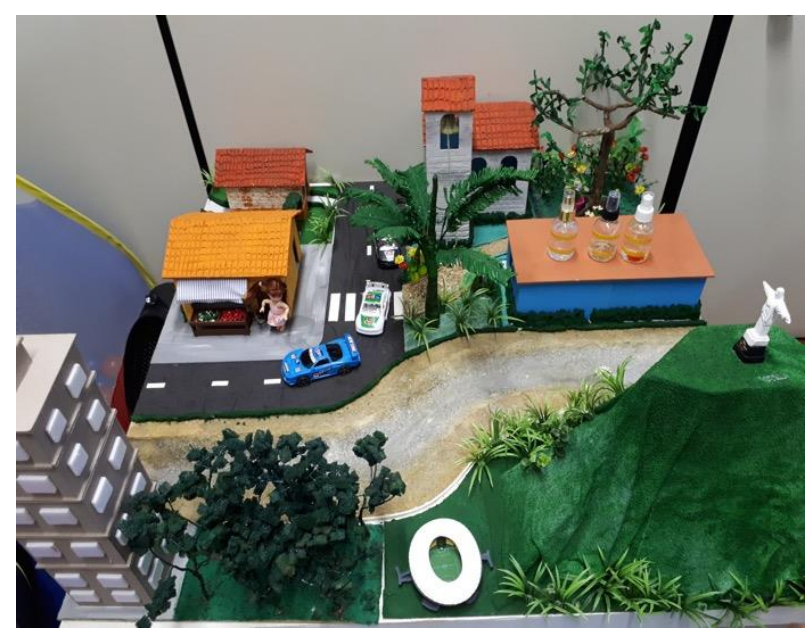

Figura 1. Maquete multissensorial "Locais"

O campo conceitual "Formas e Cores" foi integralmente adaptado, sendo necessário a adoção de processos diferentes para os dois grupos de figuras (ver Figura 2). As seis cores foram substituídas por seis novos vocábulos - liso, áspero, grande, pequeno, vazio e cheio.

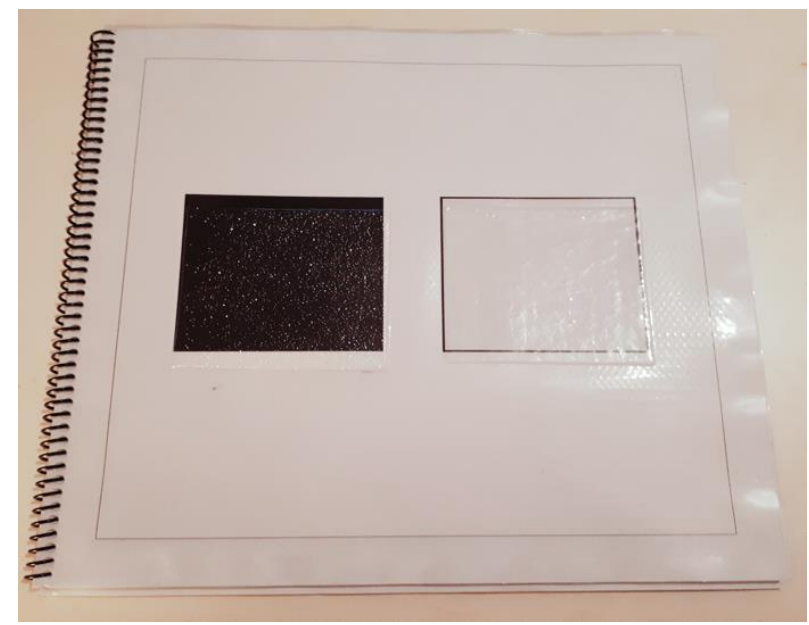

Figura 2. Prova Vocabulário, página do Livro "Formas e Opostos" referente aos vocábulos "Liso e Áspero"

As formas, quadrado, triângulo, circulo e retângulo foram adaptadas para o modelo tátil.

Para versão adaptada, elaboramos o livro "Formas e Opostos" com as figuras em thermofon. Este material possibilita a utilização do mesmo material tanto por crianças cegas quanto por crianças com baixa visão. Há uma fina película plástica com o mesmo conteúdo em tinta, porém tátil.

Cabe ressaltar que as substituições nos seis vocábulos referentes às cores, também foram substituídos para o grupo de crianças com baixa visão.

\section{Crianças com baixa visão}

A partir da aplicação das Provas de Fonologia Nomeação e Vocabulário no grupo das crianças com baixa visão, todas as figuras originais do teste foram catalogadas da seguinte forma: figuras nomeadas corretamente e figuras nomeadas incorretamente ou não nomeadas.
Este inventário pré-adaptação possibilitou verificar que 119 das figuras foram nomeadas incorretamente ou não foram nomeadas por $80 \%$ das crianças da amostra, mesmo asseguradas as acomodações ambientais e uso de recursos ópticos e recursos não ópticos.

Ou seja, 87\% das figuras demandavam adaptação considerando os princípios do design universal para pessoas com baixa visão (Allman, 2009), que inclui: adaptação de cor, sombreamento, contraste, tamanho, traço e outros.

Após esse levantamento, as figuras foram adaptadas, conforme exemplos a seguir:

- Figura "Cachorro": na figura original, a cor do cachorro não favorece a diferenciação do fundo branco (ver Figura 3).

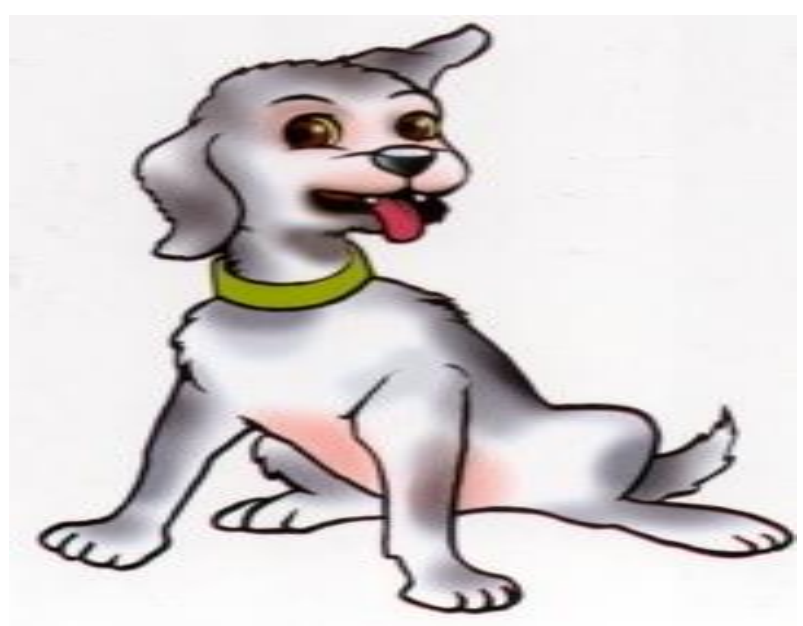

Figura 3. Figura original "cachorro"

No formato adaptado cada figura foi elaborada tendo em vista uma correta e rápida visualização figura/fundo. Neste caso, a cor do cachorro deve oferecer um bom contraste com o fundo (ver Figura 4).

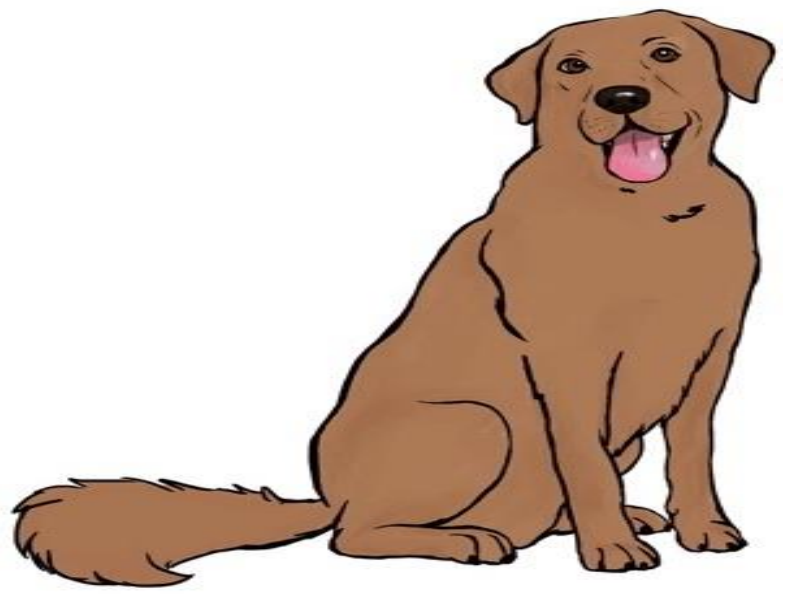

Figura 4. Figura adaptada "cachorro"

Verificou-se, após a adaptação que o percentual de designação por vocábulo usual (DVU) passa de 2 acertos para 4, ou seja, apenas uma criança utilizou o processo de substituição (onomatopéia) incorreta. 
O caso da figura "prato" (ver Figura 5), 4 crianças nomearam incorretamente, verbalizando o vocábulo "piscina".

Quando questionadas, as crianças sinalizaram que a cor azul era água e o formato era de piscina.

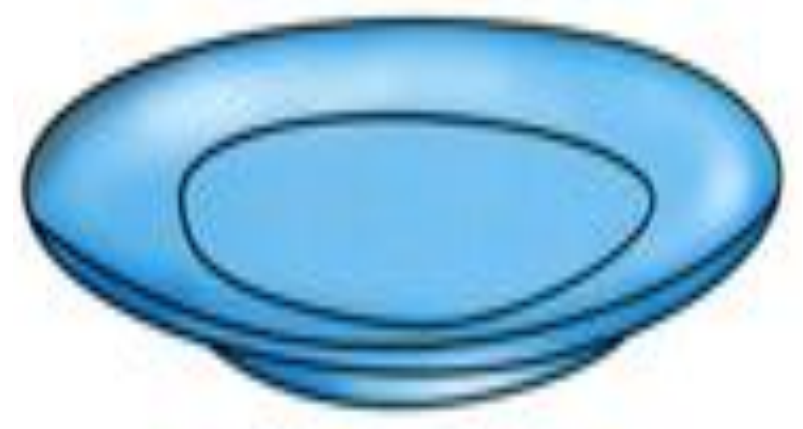

Figura 5. Figura original "prato"

Logo, na figura adaptada (ver Figura 6), procedemos a alteração principalmente na mudança nas cores, o que viabilizou um salto de 1 para 4 no percentual de designação por vocábulo usual (DVU).

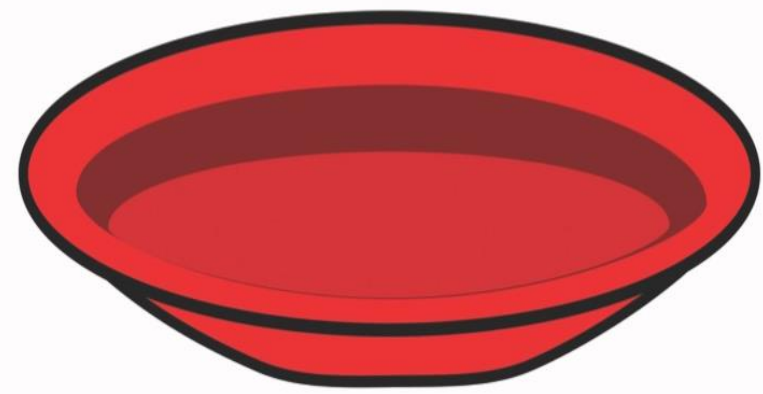

Figura 6. Figura adaptada "prato"

Outro fator importante verificado após a adaptação das figuras é a melhoria do desempenho quanto ao tempo de aplicação das provas. Houve uma otimização do tempo de rastreio e obtenção da nomeação das figuras.

\section{Adaptação nos procedimentos de aplicação, ambiente} e tempo de execução das provas

\section{Crianças cegas}

- Prova Nomeação - Fonologia e Prova de Vocabulário: como já sinalizado, todas as figuras do fichário original foram substituídas por outros recursos acessíveis a crianças cegas, isto é, substitui-se o estímulo visual por estímulos táteis, auditivos e olfativos. A partir dessa substituição as seguintes adaptações foram elencadas:

a) procedimentos de aplicação: o examinador deve apresentar os estímulos e incentivar / auxiliar a criança na exploração, especialmente aquelas com idades entre os 3 e 5 anos; deve-se estimular a utilização dos sentidos remanescentes, encorajando a criança a cheirar, pesquisar cada estímulo;

b) ambiente: os estímulos devem ser organizados por prova / campo conceitual. Para aplicação da Prova de
Vocabulário, a maquete multissensorial "Locais" deve ser preparada com antecedência pelo examinador, colocar água para o "rio" e a essência no "jardim" e na "floresta". Após a preparação, o examinador deverá primeiramente apresentar a maquete à criança, instruindo a exploração e posteriormente iniciar a prova;

c) tempo de execução: deve-se respeitar o tempo que cada criança necessita para de rastreio manual e exploração de cada estímulo. De modo geral, para a aplicação da Prova de Vocabulário, que possui 9 campos conceituais, foram necessários quatro dias com sessões de aproximadamente 90 minutos.

- Prova de Pragmática (gravação em vídeo de trinta minutos de interação entre criança e adulto): o ambiente deve ser apresentado à criança, bem como os materiais e a posição do mobiliário. Os estímulos que serão utilizados durante a prova devem ser disponibilizados de modo que a criança possa acessá-los de forma independente.

Esta adaptação na aplicação da prova da possibilita que a performance da criança não seja afetada, por exemplo pela insegurança quanto a movimentação espacial.

\section{Crianças com baixa visão}

- Prova de Nomeação - Fonologia e Prova de Vocabulário: além da adaptação das figuras, optou-se por utilizar um tablet para apresentação das figuras.

Este formato possibilita vários recursos de acessibilidade como adequação imediata e individualizada de luminosidade, melhoria de contraste, viabiliza a aproximação e distanciamento para melhor visualização dos detalhes de cada figura.

As adaptações para o grupo de crianças com baixa visão foram:

a) procedimentos de aplicação: antes de iniciar o teste deve-se verificar as preferências da criança quanto a iluminação, posicionamento do material, postura corporal, orientar o rastreio das figuras;

b) ambiente: o espaço destinado para a aplicação do teste deve estar equipado com luminárias que possam ser posicionadas de acordo com a necessidade de cada criança. O uso da prancha para apoiar o material é imprescindível, evitando assim que a criança tenha uma postura inadequada, podendo causar desconforto ou falta de atenção ao longo do teste;

c) tempo de execução: deve-se respeitar o tempo que cada criança necessita para de rastreio visual de cada figura. O tempo de aplicação das duas provas que possuem figuras - Prova de Nomeação (Fonologia) e Prova de Vocabulário teve duração em média 90 minutos.

\section{Discussão}

Os resultados obtidos a partir da versão adaptada do $\mathrm{ABFW}$, demonstraram um bom desempenho das crianças em termos de acesso às informações demandadas na aplicação do teste.

As adaptações viabilizaram a obtenção de dados objetivos para a determinação do perfil linguístico de crianças com DV, sugerindo que as modificações atenderam às equivalências necessárias para sua aplicação em crianças cegas e com baixa visão. 
O ABFW adaptado revelou-se eficiente de acordo com as especificidades e demandas de aplicação em crianças com DV. Adicionalmente importa ressaltar que o caráter lúdico presente no formato original do teste é igualmente sensível no formato adaptado. Assim, o ABFW reúne os requisitos para ser utilizado na avaliação de crianças com deficiência visual.

\section{Referências}

Andrade, C. R. F.; Béfi-Lopes, D. M.; Fernandes, F. D. M.; Wertzner, W. H. (2011). ABFW: Teste de linguagem infantil nas áreas de Fonologia, Vocabulário, Fluência e Pragmática. (2a ed. rev, ampl. e atual.). Barueri (SP):Pró-Fono.

Allman, C. (2009). Making Tests Accessible for Students with Visual Impairments: A Guide for Test Publishers, Test Developers, and State Assessment Personnel. (4th edition.) Louisville, KY: American Printing House for the Blind.

Bishop, V.E. (1998). Infants and toddlers with visual impairment. Texas School for the Blind and Visually Impaired. Recuperado de http://www.tsbvi.edu/infantintro

Mosca, R., Kritzinger, A., \& van der Linde, J. (2015). Language and communication development in preschool children with visual impairment: A systematic review. South African Journal Of Communication Disorders, 62(1), 10 pages. doi:10.4102/sajcd.v62i1.119

Mcconachie, H.R. \& Moore, V.(1994). Early expressive language of severely visually impaired children. Developmental Medicine \& Child Neurology, v. 36, n. 3, (pp. 230-240).

Pérez-Pereira,M. (2014). Contrasting views on the pragmatic abilities of blind children. Enfance, 2014, (pp 73-88.) doi:10.4074/S0013754514001062.

Rigolet, S. A. (2006). Para uma aquisição precoce e optimizada da linguagem: Linhas de orientação para crianças até 6 anos. (2a ed. rev, ampl.). Porto: Porto Editora.

Sim-Sim, I. (1998). Desenvolvimento da linguagem. Lisboa: Universidade Aberta.

Silver, M. A. (2003). Speech and language assessment. In: Goodman, S. A.; Wittenstein, S. H. (Eds.). Collaborative assessment: Working with students who are blind or visually impaired, including those with additional disabilities. ( pp. 196-236), New York: American Foundation for the Blind.

Vaan, G. D., Vervloed, M. P. J., Knoors, H., \& Verhoeven, L. (2013). Autism Spectrum Disorders in People with Sensory and Intellectual Disabilities Symptom Overlap and Differentiating Characteristics. In M. Fitzgerald (Ed.), Recent Advances in Autism Spectrum Disorders - Volume I. InTech. doi: $10.5772 / 53714$. 